\title{
Permanent Magnet Synchronous Motor Winding Fault Simulation and Diagnosis
}

\author{
Enhui Liu ${ }^{1}$, Guangxing Niu ${ }^{2}$, Shijie Tang ${ }^{3}$, Bin Zhang ${ }^{4}$, Jesse Williams ${ }^{5}$, Rodney Martin ${ }^{6}$, and Craig Moore ${ }^{7}$ \\ 1,2,3,4 University of South Carolina, Columbia, South Carolina, 29208, USA \\ eliu@email.sc.edu \\ gniu@email.sc.edu \\ shijie@email.sc.edu \\ zhangbin@cec.edu \\ ${ }^{5}$ Global Technology Connection, Atlanta, GA, 30318, USA \\ jwilliams@globaltechinc.com \\ ${ }^{6}$ NASA Ames Research Center, Moffett Field, CA, 94035, USA \\ rodney.martin@nasa.gov \\ ${ }^{7}$ NASA Marshall Space Flight Center, Huntsville, AL, 35811, USA \\ craig.e.moore@nasa.gov
}

\begin{abstract}
This paper presents the theory, simulation, diagnosis and prognosis evaluation of an anomaly detector for Permanent Magnet Synchronous Motor (PMSM) stator winding insulation faults. Physics-of-failure mechanisms are used to develop the PMSM model and its insulation fault model. Then, the diagnostic features are identified using Hilbert transforms based on artificial data acquired from the simulation results of different degree of the stator winding insulation faults. Next, the diagnosis and prognosis routine pass the diagnostic features to the Extended Kalman Filter (EKF) based on Bayesian estimation theory. Finally, the real-time diagnosis and prognosis of an anomaly detector for PMSM stator winding insulation faults are performed using Simulink. Simulation results are presented to demonstrate the effectiveness of the proposed method.
\end{abstract}

\section{INTRODUCTION}

Compared with the traditional scheduled maintenance and preventative maintenance, condition-based maintenance is a topic of growing interest in improving the reliability of target systems (Singleton, Strangas, \& Aviyente, 2014). Among the procedure of the condition based maintenance, it is of great importance to acquire online real-time data, develop models, and design algorithms that can efficiently and effectively detect faults and predict the remaining useful life (RUL) of failing components (Brown et al., 2009). The concept of RUL,

\footnotetext{
Enhui Liu et al. This is an open-access article distributed under the terms of the Creative Commons Attribution 3.0 United States License, which permits unrestricted use, distribution, and reproduction in any medium, provided the original author and source are credited.
}

which is defined as the time interval from current time to the time instant when the system fails to deliver its service, has been widely used in the community of prognostics and health management (PHM) (Si, Wang, Hu, \& Zhou, 2011; Zio \& Peloni, 2011; X. Zhang et al., 2005; Jardine, Lin, \& Banjevic, 2006; M. E. Orchard \& Vachtsevanos, 2007) for enabling optimal maintenance and enhancing system safety.

With the development of technology, PMSM has been widely used in industries because of its advantages of high efficiency, high output to volume ration, high power to weight ratio, low noise emissions, simple construction, easy maintenance, high-speed operation, and precise torque control (Yang, Ye, Zhou, et al., 2014). Previous research works indicated that winding insulation fault is one of the primary motor failure mode (Yang et al., 2014). The leading causes of stator winding insulation faults are high temperature and overloading. Winding insulation faults can lead to fluctuations in torque, imbalance, current harmonics, overheating, and vibration. Without timely detection and correction actions, it can rapidly propagate to more stator winding turns and then may cause demagnetization, power loss or damage of PMSM.

For PMSM fault diagnosis, the data, such as voltage and current, need to be collected from PMSM and analyzed to extract features or fault indicators. Data processing methods usually detect faults by comparing the measured data/simulated data between healthy PMSM and faulty PMSM. Some widely used data processing methods include Short-time Fourier transform (STFT), wavelet transform (WT), Wigner-Ville distribution (WVD) and Empirical Mode Decomposition (EMD). Each method has its own advantages and limitations. 
For example, STFT needs to select a suitable window (Hamming, Gaussian, etc.) to match with the specific frequency content of the signals. Moreover, STFT is not suitable to process non-stationary signals. WT can preserve spectral information efficiently but cannot express spatial characteristics well. Moreover, an important limitation of the WT is its nonadaptive nature (Y. Zhang, 2006). WVD is similar to STFT and is not suitable for unsteady signals. EMD decomposes the signal into a set of intrinsic mode functions (IMFs) and a residual. Although EMD is effective to extract features, IMFs are not related to frequency. Hilbert transform is a simple and adaptive technique for PMSM fault diagnosis. Through Hilbert spectrum, full frequency-time energy distribution of the signal can be obtained. Moreover, Hilbert spectrum has no cross frequencies, and has no boundary problems (Yang et al., 2014).

A fault diagnosis procedure includes the tasks of fault detection and assessment of its severity. In this sense, an EKF based diagnosis framework is proposed in this research to achieve these goals. it is assumed that the fault dynamics are nonlinear Gaussian Markov processes.

In previous research work, the stator insulation fault injection is a type of step signal, which can not reflect the actual situation as the stator insulation fault follows a certain degradation law in the motor operation (Brown et al., 2009). In this paper, to make the motor operates in a more realistic situation, the degradation of the stator insulation is modeled as a dynamic process. In this way, the identification of diagnostic feature and the real-time performance of diagnosis and prognosis can be more reliable. Moreover, this paper aims to propose a comprehensive approach to develop, analyze, and validate extended Kalman filter (EKF) based fault diagnosis and failure prognosis of PMSM stator winding insulation faults. The proposed method has an integrated framework that adopts Bayesian estimation methods and simulation data acquired through the Simulink model of PMSM with motor stator winding insulation faults being injected. Since the fault dynamics are nonlinear, it is necessary to develop methods that can accommodate nonlinear systems. For this purpose, a physics-based PMSM state-space model is developed and stator winding insulation fault condition indicators are extracted from simulation data based on Hilbert transform. The proposed framework employs the PMSM model and EKF to estimate the state of motor winding fault in real time, which is given by probability density function (PDF) (M. Orchard, Kacprzynski, Goebel, Saha, \& Vachtsevanos, 2008).

In this paper, a PMSM model established in Simulink is selected as a prototype testbed for proof-of-concept. The paper is organized as follows: Section 2 presents the theory of operation for a PMSM along with stator winding insulation faults; Section 3 discusses the simulation results obtained from a test conducted with PMSM with dynamic changing stator wind- ing insulation faults. Primary feature is extracted based on the Hilbert transform. Section 4 provides the theory and simulation results of fault diagnosis and prognosis based on EKF to validate the proposed model and approach; Section 5 summarizes the results of the study and provides concluding remarks.

\section{PMSM Modeling AND FEATURE EXTRACTION}

\subsection{Three Phase PM Synchronous Machine Model}

Fig. 1 shows the equivalent circuit diagram of a three-phase wye-connected PMSM [Note: the stator windings are displaced evenly with a degree of 120 degree]. In this figure, $U_{a s}, U_{b s}$ and $U_{c s}$ are input voltage to phase a, b and c of the motor, $L_{a a}, L_{b b}$ and $L_{c c}$ are selr-inductance of phase a, $\mathrm{b}$ and c, $r_{a a}, r_{b b}$ and $r_{c c}$ are resistance of phase a, b and c, $L_{a b}, L_{a c}$ and $L_{b c}$ are mutual inductance between phases, $i_{a s}, i_{b s}$ and $i_{c s}$ are stator current of phase a, b and c, $\psi_{a s}, \psi_{b s}$ and $\psi_{c s}$ are flux linkage of phase $a, b$ and $c$, respectively.

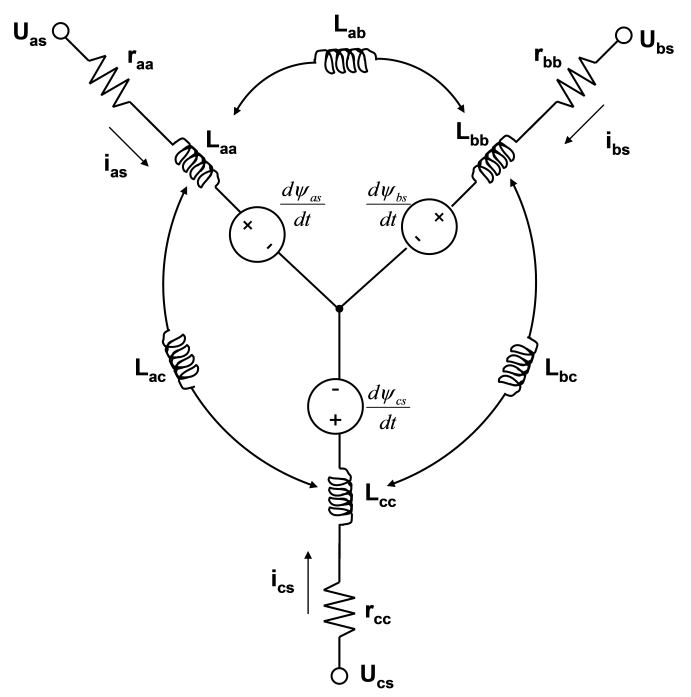

Figure 1. Three-phase wye-connected permanent-magnet synchronous motor.

With this equivalent circuit and Kirchoff's law, a set of differential equations can be obtained as:

$$
\begin{aligned}
\mathbf{U}_{\mathrm{abcs}} & =\mathbf{r}_{\mathrm{abcs}} \mathbf{i}_{\mathrm{abcs}}+\mathbf{L}_{\mathrm{abcs}} \frac{d}{d t} \mathbf{i}_{\mathrm{abcs}}+\frac{d}{d t}\left[\mathbf{\Psi}_{\mathrm{abcs}}\right], \\
{\left[\begin{array}{c}
U_{a s} \\
U_{b s} \\
U_{c s}
\end{array}\right] } & =\left[\begin{array}{ccc}
r_{a a} & 0 & 0 \\
0 & r_{b b} & 0 \\
0 & 0 & r_{c c}
\end{array}\right]\left[\begin{array}{c}
i_{a s} \\
i_{b s} \\
i_{c s}
\end{array}\right] \\
+ & {\left[\begin{array}{ccc}
L_{a a} & L_{b a} & L_{c a} \\
L_{a b} & L_{b b} & L_{c b} \\
L_{a c} & L_{b c} & L_{c c}
\end{array}\right] \frac{d}{d t}\left[\begin{array}{c}
i_{a s} \\
i_{b s} \\
i_{c s}
\end{array}\right] } \\
+ & \frac{d}{d t}\left[\begin{array}{c}
\psi_{a s} \\
\psi_{b s} \\
\psi_{c s}
\end{array}\right] .
\end{aligned}
$$


where $L_{a b}=L_{b a}, L_{a c}=L_{c a}, L_{b c}=L_{c b}$

These equations describe the relationships between the stator voltages $\left(U_{a s}, U_{b s}\right.$ and $\left.U_{c s}\right)$ with stator current $\left(i_{a s}, i_{b s}\right.$ and $\left.i_{c s}\right)$, flux linkage $\left(\psi_{a s}, \psi_{b s}\right.$ and $\left.\psi_{c s}\right)$, and resistance $\left(r_{a a}, r_{b b}\right.$ and $r_{c c}$ ) for each winding (Lyshevski, Skormin, \& Colgren, 2002).

Note that the third term in Eq. (1) can be expanded in terms of the winding inductance matrix $\mathbf{L}_{\mathbf{a} b \mathbf{c}}$ and magnetic flux linkage vector $\Psi_{m}$ as (Brown et al., 2009):

$$
\begin{aligned}
\boldsymbol{\Psi}_{\mathrm{abcs}} & =\mathbf{L}_{\mathrm{abcs}} \mathbf{i}_{\mathrm{abcs}}+\boldsymbol{\Psi}_{\mathrm{m}} \\
{\left[\begin{array}{c}
\psi_{a s} \\
\psi_{b s} \\
\psi_{c s}
\end{array}\right] } & =\left[\begin{array}{ccc}
L_{a a} & 0 & 0 \\
0 & L_{b b} & 0 \\
0 & 0 & L_{c c}
\end{array}\right]\left[\begin{array}{l}
i_{a s} \\
i_{b s} \\
i_{c s}
\end{array}\right] \\
& +\left[\begin{array}{c}
\psi_{a m} \\
\psi_{b m} \\
\psi_{c m}
\end{array}\right]
\end{aligned}
$$

where $\psi_{a m}, \psi_{b m}$ and $\psi_{c m}$ are magnetic flux linkage of phase $\mathrm{a}, \mathrm{b}$ and $\mathrm{c}$ respectively. Flux linkages at the stator windings due to the permanent magnets on the rotor are given as (Brown et al., 2009):

$$
\Psi_{\mathrm{m}}=\left[\begin{array}{c}
\psi_{\mathrm{am}} \\
\psi_{\mathrm{bm}} \\
\psi_{\mathrm{cm}}
\end{array}\right]=\psi_{\mathrm{m}}\left[\begin{array}{c}
\sin (\theta) \\
\sin \left(\theta-\frac{2 \pi}{3}\right) \\
\sin \left(\theta+\frac{2 \pi}{3}\right)
\end{array}\right]
$$

where $\theta$ denotes the angle between rotor and stator.

The torque $T_{e}$ can be described by first developing an analytical expression for the energy stored in the magnetic flux $\psi_{m}$, given in Eq. (4), in which $P$ is the number of magnetic poles (Lyshevski et al., 2002).

$$
W_{m}=\frac{P}{2}\left\{\frac{1}{2} \mathbf{i}_{\text {abcs }}^{T} \mathbf{L}_{\text {abcs }} \mathbf{i}_{\text {abcs }}+\mathbf{i}_{\text {abcs }}^{T} \mathbf{\Psi}_{\mathbf{m}}\right\}
$$

Then the motor torque is computed by taking the derivative of $W_{m}$ with respect to the rotor position $\theta$, which leads to torque expression (Lyshevski et al., 2002):

$$
T_{e}=\frac{P}{2}\left\{\frac{1}{2} \mathbf{i}_{\mathrm{acss}}^{T} \frac{d\left(\mathbf{L}_{\mathrm{abcs}}\right)}{d \theta} \mathbf{i}_{\mathrm{abcs}}+\mathbf{i}_{\mathrm{abcs}}^{T} \frac{d\left(\boldsymbol{\psi}_{\mathrm{m}}\right)}{d \theta}\right\}
$$

\subsection{Stator Winding Insulation Fault Model}

The primary failure mechanisms for the PMSM includes stator winding insulation fault, turn-to-phase short circuits and open circuits. A stator winding insulation fault can result in a three-phase impedance imbalance in the stator windings, which will lead to asymmetries in the phase currents, phase voltage, increased harmonic generation, torque fluctuation, and some other performance degradations (Chang, Cocquempot, \& Christophe, 2003; Penman, Sedding, Lloyd, \& Fink, 1994). In this study, we only focus on a single stator winding insulation fault for PMSM.

Fig. 2 shows a schematic that represents a winding fault for a single winding, in which $L_{s}, R_{s}$ and $U_{s}$ represent the total winding inductance, resistance and back-emf voltage of the winding, $N, k$ and $R_{f}$ represent the number of total winding turns, number of winding turns between the fault, and the resistance of the insulation fault, respectively. Note that $k=0$ indicates a normal condition.

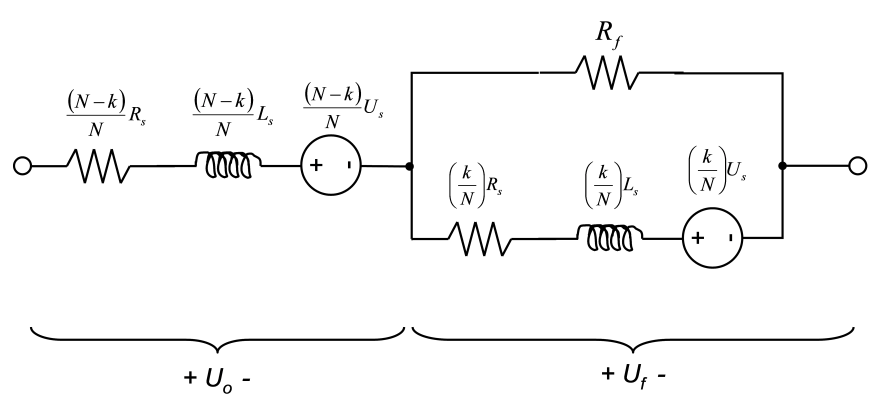

Figure 2. Schematic of insulation fault model.

The circuit network on the right side of Fig. 2 can be reduced to a single resistor, inductor and voltage source, as illustrated below in Fig. 3, by applying the Thevenin circuit transformation.

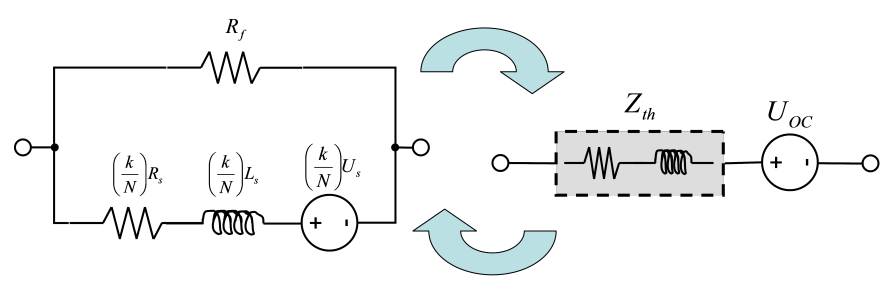

Figure 3. Thevenin circuit transformation of the winding fault model.

With some mathmatical operations, simplified expressions for $R_{s}^{f}, L_{s}^{f}$, and $\psi_{s}^{f}$ can be obtained in terms of $L_{s}, R_{s}, \psi_{s}$ and $w_{f}$ gien as.

$$
\left[\begin{array}{lll}
R_{s}^{f}(t) & L_{s}^{f}(t) & \psi_{s}^{f}(t)
\end{array}\right]^{T} \approx w_{f}(t)\left[\begin{array}{lll}
R_{s} & L_{s} & \psi_{s}
\end{array}\right]^{T}
$$

where $w_{f}$ is fault index, which is inversely proportional to the dimension of the stator winding insulation fault and is expressed as:

$$
w_{f}\left(R_{f}, R_{s}, k, N\right)=1-\frac{k}{N}\left(1-\left[1+\frac{k}{N}\left(\frac{R_{s}}{R_{f}}\right)\right]^{-2}\right)
$$

where $0 \leq w_{f} \leq 1$. 
It shows that the fault index $w_{f}$ depends on the insulation fault resistance $R_{\mathrm{f}}$, stator resistance $R_{\mathrm{S}}$, number of effected turns $k$, and total number of turns per winding $N$. Define the conditional fault indexes for three phases $(\mathrm{a}, \mathrm{b}$ and $\mathrm{c})$ as $w_{a}, w_{b}$ and $w_{c}$. When the stator winding insulation faults are injected, the relevant parameters of the PMSM need to be modified by the conditional fault indexes.

\subsection{Feature Selection and Extraction}

By combining the subsystems developed in the previous section, a complete three phase PMSM model is established in Simulink as shown in Fig. 4

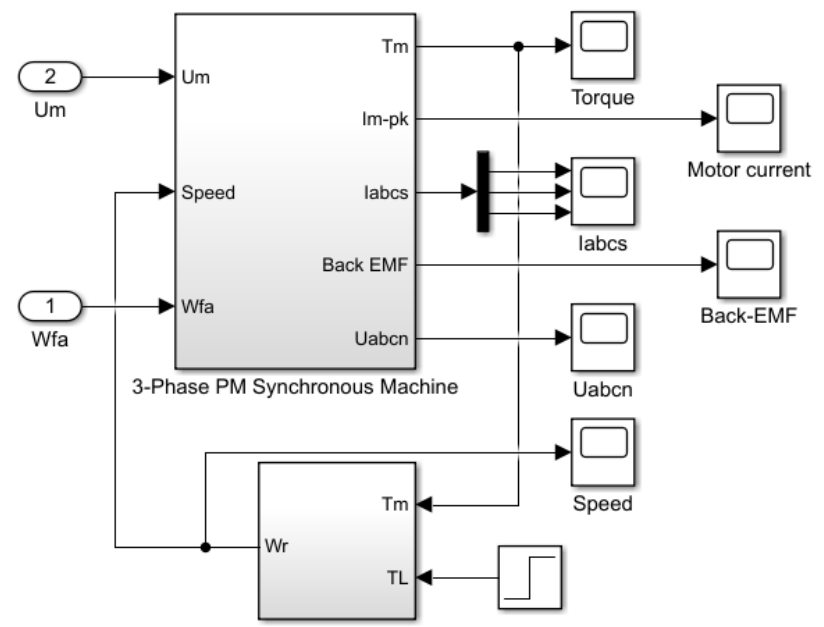

Figure 4. Advanced 3-phase PM machine model.

The PMSM Simulink model is used to simulate the fault scenarios. A stator winding insulation fault is injected to phase $\mathrm{A}$ at the 5th second and continuously simulated with phase A winding fault index $w_{\mathrm{a}}$ decreasing. The changes of stator winding insulation fault is given as Fig. 5. Figs. 6 and 7 shows the simulation results without and with insulation fault on phase A winding. From these figures, it is clear that the phase currents become asymmetric under fault conditions.

With the simulated data shown in Figs. 6 and 7, features or condition indicators need to be extracted from them to detect and isolate winding faults. This section develops feature extraction methods from simulated phase current $i_{i s}(t)$ by Hilbert transform to detect asymmetries caused by winding insulation faults. Here the subscript index $i$ refers to the phase current of the $i$ th phase winding and $s$ indicates steady state.

Hilbert Transform is a convolution between the Hilbert transformer $1 /(\pi t)$ and an original phase current signal $i_{i s}(t)$. This results in phase shifting from the original phase current signal $i_{i s}(t)$ by $\pi / 2$ radians. The Hilbert transform $\hat{i}_{i s}(t)$ of original signal $i_{i s}(t)$ is defined for all $t$ by (Henrici, 1993),

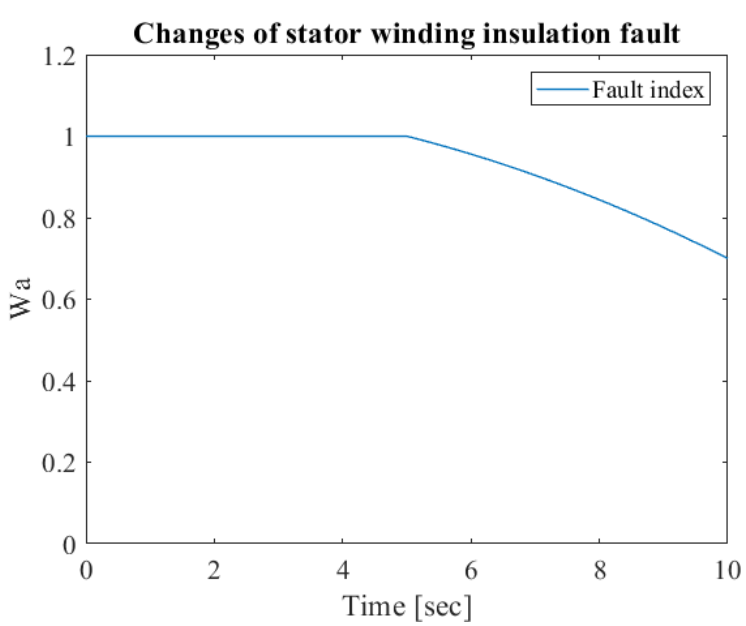

Figure 5. Simulated phase currents for no fault .

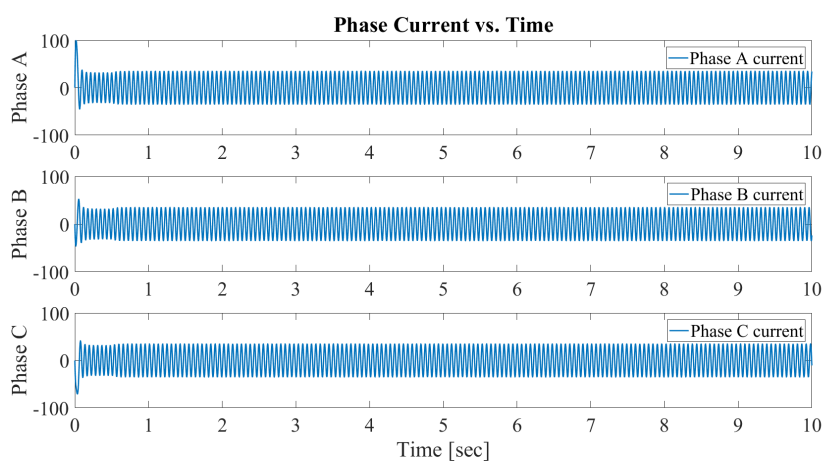

Figure 6. Simulated phase currents for no fault .

$$
\hat{i}_{i s}(t)=\frac{1}{\pi} P \int_{-\infty}^{\infty} \frac{i_{i s}(\tau)}{t-\tau} d \tau
$$

The Hilbert transform can be used to create an analytic signal $z(t)$ from a real signal $i_{i s}(t)$,

$$
z(t)=i_{i s}(t)+j \hat{i}_{i s}(t)
$$

The signal $z(t)$ can be described as a rotating vector,

$$
Z(t)=A(t) e^{i_{i s} \varphi(t)}
$$

where $A(t)$ is amplitude and $\varphi(t)$ is the phase, which are given as,

$$
\begin{aligned}
& A(t)=\sqrt{i_{i s}^{2}(t)+\hat{i}_{i s}^{2}(t)}, \\
& \varphi(t)=\arctan \left[\hat{i}_{i s}(t) / i_{i s}(t)\right] .
\end{aligned}
$$




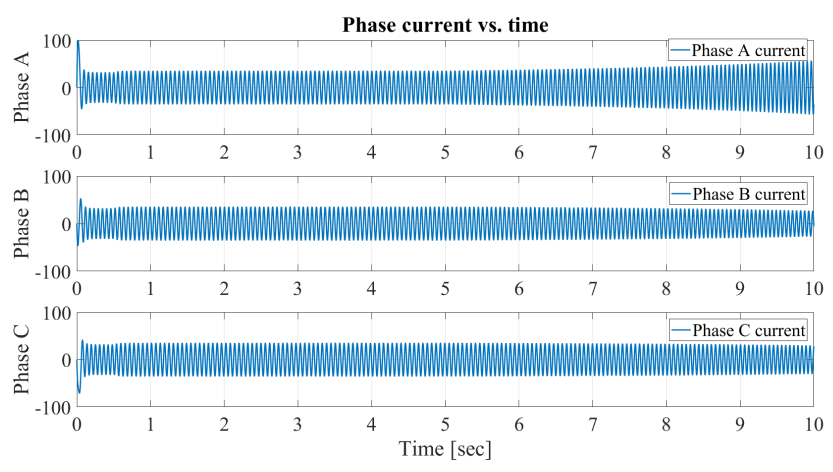

Figure 7. Simulated phase currents for stator winding insulation fault with fault index $w_{a}$ being decreased.

Then for a real signal $i_{i s}(t)=A_{0} \cos \left(\omega_{0} t+\varphi_{0}\right)$, its Hilbert transform is given as:

$$
\hat{i}_{i s}=A_{0} \sin \left(\omega_{0} t+\varphi_{0}\right)
$$

From Eqs. (8) and (11), we can see that the analytical signal $z(t)$ 's amplitude $A(t)$ is a constant and is phase invariant i.e.,

$$
A(t)=A_{0}
$$

The above analysis shows that for a motor current signal given in sinusoidal form, when the motor is working under constant condition, the motor current signal has constant amplitude and frequency, $\left|i_{i s}(t)+\hat{i}_{i s}(t)\right|$ is phase invariant. This result can be extended to time-varying three phase current signals to extract features for fault detection. In the case of a motor operates without fault, $\left|i_{i s}(t)+\hat{i}_{i s}(t)\right|$ should be phaseinvariant. On the contrary, when the motor operates with a fault, the amplitude of $\left|i_{i s}(t)+\hat{i}_{i s}(t)\right|$ will change. Therefore, we can evaluate the winding turn-to-turn fault dimension of motor based on the change of $\left|i_{i s}(t)+\hat{i}_{i s}(t)\right|$.

In this paper, the standard deviation of the average amplitude of each phase current (a,b, c) over a finite time interval $T$, denoted as $i_{p}(t, T)$ is used to describe variations in winding symmetry (Brown et al., 2009).

$$
i_{p}(t, T)=\operatorname{std}_{i \in a, b, c}\left[\operatorname{avg}_{t \in(0, T)}\left|i_{i s}(t)+\hat{i}_{i s}(t)\right|\right]
$$

With the above analysis, features can be extracted to indicate the condition of faults. With the simulation signal from Figs. 6 and 7, Fig. 8 shows the feature value for fault-free case and Fig. 9 shows the feature value when insulation fault is injected and insulation condition continuously degrades.

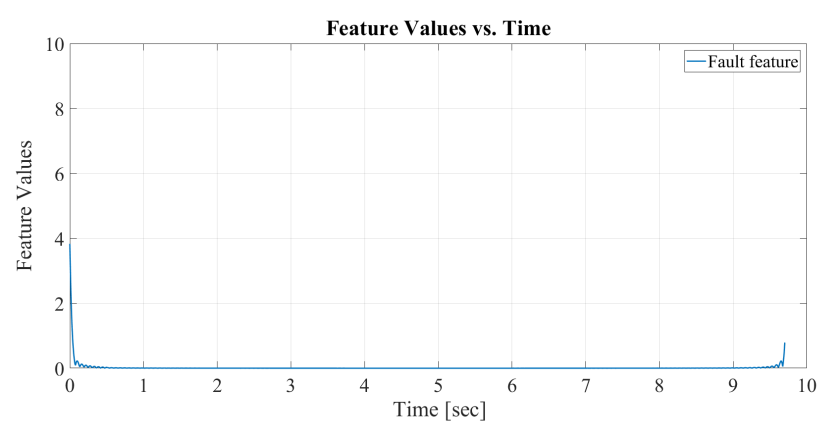

Figure 8. Simulated feature values derived from phase currents with no fault.

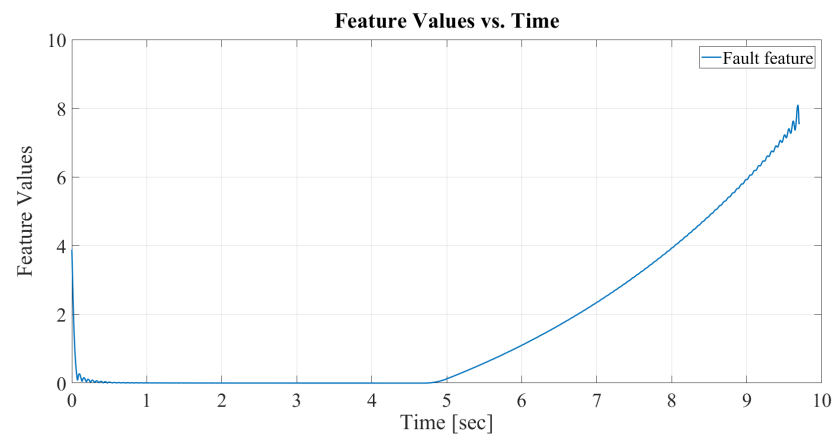

Figure 9. Simulated feature values derived from phase currents for turn-to-turn winding insulation fault with fault dimension being increased.

\section{Anomaly Detection and Prognosis}

Kalman filtering is a recursive algorithm that estimates the true state of a system based on noisy measurements. Kalman filter (KF) has been used in many applications involving navigation (Geng \& Wang, 2008), online system identification (Wu \& Smyth, 2007), tracking and fault prognosis (Mizumoto, Takahashi, Ogata, \& Okuno, 2012). EKF is an extension for nonlinear system dynamics. In EKF, the system model is linearized around the current state to obtain a priori state estimate. Then when the measurement becomes available, it is used to update the prior state estimate to obtain a posteriori estimation. Mathematically, it is assumed that the motor winding fault dynamics can be described by the following nonlinear systems.

$$
\begin{gathered}
x_{k+1}=f\left(x_{k}, u_{k}\right)+w_{k} \\
y_{k}=h\left(x_{k}, u_{k}\right)+v_{k}
\end{gathered}
$$

where Eq. (14) describes the state transition, and Eq. (15) is the observation model that describes the relationship between state and measurements. In Eqs. (14) and (15), $w_{k}$ and $v_{k}$ are 
noise terms that are assumed to be Gaussian noise, with zero mean and known covariance matrices $Q$ and $R$ respectively.

The process of the EKF algorithm can be divided into two steps, prediction and update.

Since the fault growth is non-linear process, the Jacobian of the non-linear functions $f(\cdot)$ and $h(\cdot)$, denoted as $F_{k}$ and $H_{k}$ respectively, are calculated at each time instant as:

$$
\begin{aligned}
& F_{k}=\left.\frac{\partial f\left(x_{k}, u_{k}\right)}{\partial x_{k}}\right|_{x_{k}=\hat{x}_{k+1 \mid k}} \\
& H_{k}=\left.\frac{\partial h\left(x_{k}, u_{k}\right)}{\partial x_{k}}\right|_{x_{k}=\hat{x}_{k+1 \mid k}}
\end{aligned}
$$

The prediction step is to calculate the prior estimate, which can be described by the following equations:

$$
\begin{gathered}
\hat{x}_{k+1 \mid k}=f\left(\hat{x}_{k \mid k}, u_{k}\right)+w_{k} \\
P_{k+1 \mid k}=F_{k} P_{k \mid k} F_{k}^{T}+Q_{k}
\end{gathered}
$$

Then, the Kalman gain is calculated as:

$$
K_{k+1}=P_{k+1 \mid k} H_{k+1}^{T}\left(H_{k+1} P_{k+1 k} H_{k+1}^{T}+R_{k+1}\right)^{-1}
$$

The updated state estimate is then obtained as:

$$
\hat{x}_{k+1 \mid k+1}=\hat{x}_{k+1 \mid k}+K_{k+1} z_{k+1}
$$

where $z_{k+1}$ is the measurement residual, which is given by

$$
z_{k+1}=\hat{y}_{k+1}-h\left(\hat{x}_{k+1 \mid k}, u_{k}\right)
$$

The last step is to update the covariance estimate,

$$
P_{k+1 \mid k+1}=\left(I-K_{k+1} H_{k+1}\right) P_{k+1 \mid k}
$$

To implement diagnosis and prognosis based on EKF method, a set of features generated earlier in Figs. 6 and 7 were used to develop fault growth model which is given by:

$$
y(t)=y(t-1)+p_{1} \cdot\left(p_{2} \cdot t+p_{3} \cdot t^{-2}\right)+w(t)
$$

where $t$ is time index, $p=[3.2 \mathrm{e}-5,8,1 \mathrm{e}-3]$ are parameters, $\omega(t) \sim N(0,0.02)$ is the model noise.

\section{EXPERIMENTAL RESULTS USING ARTIFI- CIAL DATA}

In fault diagnosis, data from the first 3 seconds are used to build the baseline distribution. In this project, false alarm and confidence of detection are defined as 5\% and 90\%, respectively. The baseline distribution is shown in Fig. 11(a), and the fault detection threshold (blue line) can be obtained based on the baseline pdf and false alarm rate.

When the diagnosis algorithm is conducted, the real-time pdf will be compared with the baseline pdf. Since the false alarm rate is set as $5 \%$, if $90 \%$ of the real-time pdf is beyond the fault detection threshold, we can consider that a fault is detected with $5 \%$ false alarm rate and $90 \%$ confidence. Fig. 10 shows EKF based non-linear estimation.

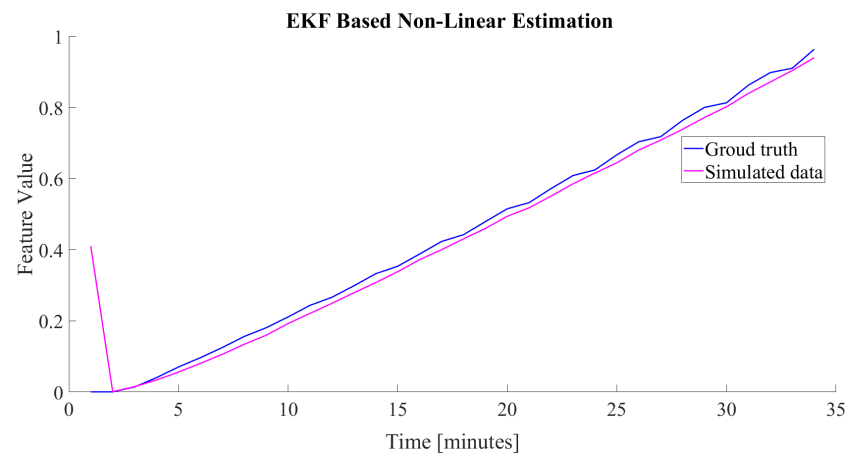

Figure 10. EKF based non-linear estimation.

The prognosis begins when the fault is detected. In this paper, the failure threshold is set to 7 , and the posterior fault state distribution from diagnosis is used as initial condition of prognosis. In fault prognosis process, the $\mu_{e k f}$, which is the estimated mean value of fault feature is only updated by previous $\mu_{e k f}$ value. The results of the fault diagnosis and prognosis algorithm are shown in Fig. 11(b).
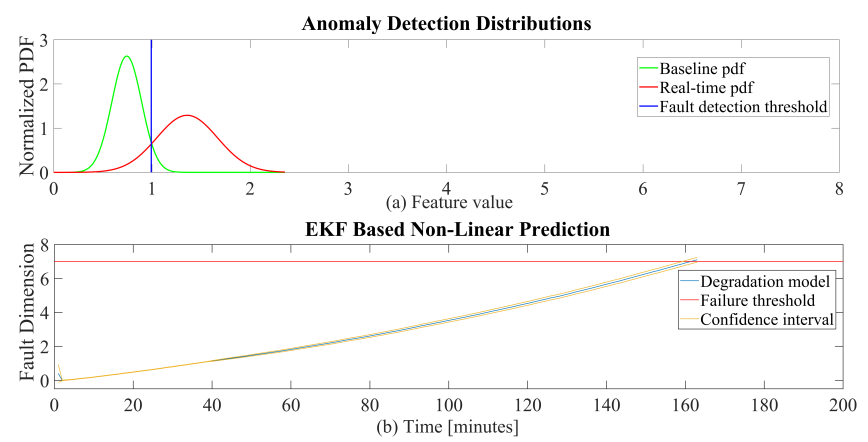

Figure 11. Results of fault diagnosis and prognosis.

To evaluate the accuracy of prognosis, $\alpha-\lambda$ metric is used, which compares the actual RUL to the predicted RUL with an $\alpha$ bounds around the actual RUL. Figure 12 shows the $\alpha-\lambda$ metric with simulated data. $\alpha-\lambda$ metric (Zheng $\&$ Fang, 2015) is applied to evaluate the performance of this prognostic evaluation as shown in Fig. 12. From Fig. 12, the prognostic algorithm performs well as its accuracy improves quickly with time within the $30 \%$ bounds. 


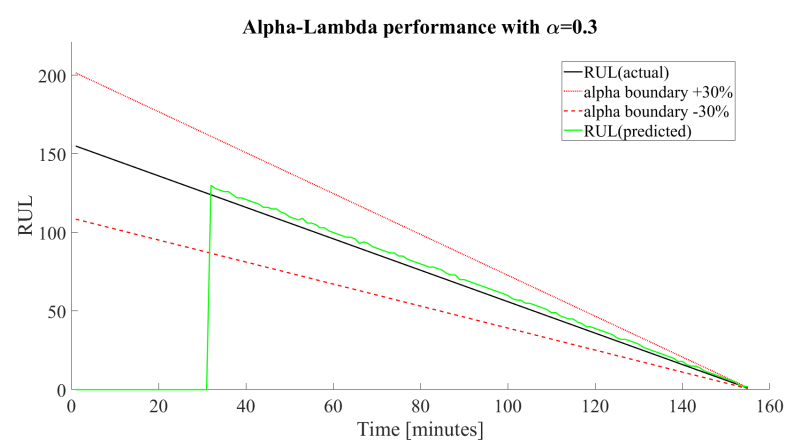

Figure 12. Alpha-Lambda performance metric using 30\% accuracy bounds.

\section{Conclusion}

In this study, EKF is implemented for fault detection and RUL estimates of PMSM. A PMSM fault model in Simulink environment is built with fault injection. The fault model is investigated in detail with stator winding insulation fault. The primary fault mode is modeled for early fault detection in the presence of faults with dimension being increased. Simulation results demonstrate the effectiveness of the proposed method for PMSM insulation fault diagnosis.

\section{NoMENCLATURE}

$\psi_{\mathrm{abcs}}$
$\psi_{\mathrm{m}}$
$r_{a a}, r_{b b}, r_{c c}$
$L_{a a}, L_{b b}, L_{c c}$
$L_{a b}, L_{a c}, L_{b c}$
$N$
$k$
$L_{S}$
$R_{s}$
$R_{s}^{f}, R_{f}$
$Z_{t h}$
$L_{s}^{f}$
$W_{f}$
$i_{\mathrm{abcs}}$
$\omega_{r}$
$T_{e}$
$\theta$
$W_{m}$
$U_{\mathrm{oc}}$
$U_{\mathrm{abcs}}$
$U_{\mathrm{o}}$
$U_{\mathrm{f}}$

Flux linkages

Max flux linkage

Single phase resistance

Self-inductance

Mutual inductance

Number of total winding turns

Number of winding turns between fault

Total winding inductance during normal conditions

Winding resistance during normal conditions

Resistance of the insulation fault

Equivalent Thevenin impedance

Self-inductance of winding with insulation fault

Fault index

Stator current

Motor speed

Torque

The angle between stator and rotor

Energy stored in magnetic flux

Open circuit voltage of fault part

Output voltage for each winding

Voltage of faultless part of the winding

Voltage of fault part of the winding

\section{REFERENCES}

Brown, D., Georgoulas, G., Bae, H., Vachtsevanos, G., Chen, R., Ho, Y., ... Schroeder, J. (2009). Particle filter based anomaly detection for aircraft actuator systems. In 2009 ieee aerospace conference (pp. 1-13).

Chang, X., Cocquempot, V., \& Christophe, C. (2003). A model of asynchronous machines for stator fault detection and isolation. IEEE transactions on industrial electronics, 50(3), 578-584.

Geng, Y., \& Wang, J. (2008). Adaptive estimation of multiple fading factors in kalman filter for navigation applications. GPS solutions, 12(4), 273-279.

Henrici, P. (1993). Applied and computational complex analysis, volume 3: Discrete fourier analysis, cauchy integrals, construction of conformal maps, univalent functions (Vol. 3). John Wiley \& Sons.

Jardine, A. K., Lin, D., \& Banjevic, D. (2006). A review on machinery diagnostics and prognostics implementing condition-based maintenance. Mechanical systems and signal processing, 20(7), 1483-1510.

Lyshevski, S. E., Skormin, V. A., \& Colgren, R. D. (2002). High-torque density integrated electromechanical flight actuators. IEEE Transactions on Aerospace and Electronic Systems, 38(1), 174-182.

Mizumoto, T., Takahashi, T., Ogata, T., \& Okuno, H. G. (2012). Adaptive pitch control for robot thereminist using unscented kalman filter. In Modern advances in intelligent systems and tools (pp. 19-24). Springer.

Orchard, M., Kacprzynski, G., Goebel, K., Saha, B., \& Vachtsevanos, G. (2008). Advances in uncertainty representation and management for particle filtering applied to prognostics. In 2008 international conference on prognostics and health management (pp. 1-6).

Orchard, M. E., \& Vachtsevanos, G. J. (2007). A particle filtering-based framework for real-time fault diagnosis and failure prognosis in a turbine engine. In 2007 mediterranean conference on control \& automation (pp. 1-6).

Penman, J., Sedding, H., Lloyd, B., \& Fink, W. (1994). Detection and location of interturn short circuits in the stator windings of operating motors. IEEE transactions on Energy conversion, 9(4), 652-658.

Si, X.-S., Wang, W., Hu, C.-H., \& Zhou, D.-H. (2011). Remaining useful life estimation-a review on the statistical data driven approaches. European journal of operational research, 213(1), 1-14.

Singleton, R. K., Strangas, E. G., \& Aviyente, S. (2014). Extended kalman filtering for remaining-useful-life estimation of bearings. IEEE Transactions on Industrial Electronics, 62(3), 1781-1790.

Wu, M., \& Smyth, A. W. (2007). Application of the unscented kalman filter for real-time nonlinear structural system identification. Structural Control and Health Monitoring: The Official Journal of the International Association for Structural Control and Monitoring and of the European Association for the Control of Structures, 14(7), 971-990. 
Yang, J., Ye, H., Zhou, W., et al. (2014). A review of permanent magnet synchronous motor fault diagnosis. In 2014 ieee conference and expo transportation electrification asia-pacific (itec asia-pacific) (pp. 1-5).

Zhang, X., Xu, R., Kwan, C., Liang, S. Y., Xie, Q., \& Haynes, L. (2005). An integrated approach to bearing fault diagnostics and prognostics. In Proceedings of the 2005, american control conference, 2005. (pp. 2750-2755).

Zhang, Y. (2006). Hilbert-huang transform and marginal spectrum for detection of bearing localized defects. In 2006 6th world congress on intelligent control and automation (Vol. 2, pp. 5457-5461).

Zheng, X., \& Fang, H. (2015). An integrated unscented kalman filter and relevance vector regression approach for lithium-ion battery remaining useful life and shortterm capacity prediction. Reliability Engineering \& System Safety, 144, 74-82.

Zio, E., \& Peloni, G. (2011). Particle filtering prognostic estimation of the remaining useful life of nonlinear components. Reliability Engineering \& System Safety, 96(3), 403-409.

\section{BIOGRAPHIES}

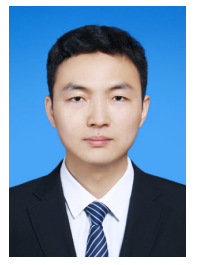

Enhui Liu received the B.A. degree from Shanddong University of Architecture,Jinan, China, in 2015, and the M.S.E. degreee from Harbin Institute of Technology, Shenzhen, China, in 2018, both in electrical engineering. He is currently working toward the Ph.D. degree in electrical engineering from the University of South Carolina, Columbia, SC, USA. His current interest focuses on diagnosis, prognosis and Health Management to improve the reliability of target systems.

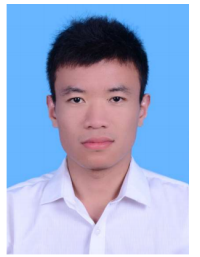

Guangxing Niu received the B.A. degree from North University of China, Taiyuan, China, in 2014, and the M.S.E. degree from Harbin Institute of Technology, Harbin, China, in 2016, both in electrical engineering. $\mathrm{He}$ is currently working toward the Ph.D. degree in electrical engineering from the University of South Carolina, Columbia, SC, USA. His current research focuses on the data-driven-based algorithms of diagnosis and prognosis for lithium ion batteries and industrial systems.

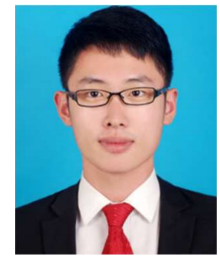

Shijie Tang received the B.E. degree in Electrical Engineering from Yanshan University, Qinhuangdao, China, in 2016. $\mathrm{He}$ is currently pursuing the Ph.D. degree in Electrical Engineering at University of South Carolina, Columbia, South Carolina, US. His current research interests are Fault Diagnosis, Prognostic and
Health Management, Machine Learning and Control Theory.

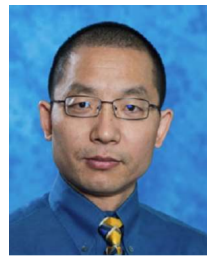

Bin Zhang received the B.E. and M.E. degrees from the Nanjing University of Science and Technology, Nanjing, China, in 1993 and 1999, respectively, and the $\mathrm{Ph} . \mathrm{D}$. degree from Nanyang Technological University, Singapore, in 2007. He was with Research and Development, General Motors, Detroit, MI, USA, Impact Technologies, Rochester, NY, USA, and the Georgia Institute of Technology, Atlanta, GA, USA. He is currently with the Department of Electrical Engineering, University of South Carolina, Columbia, SC, USA.

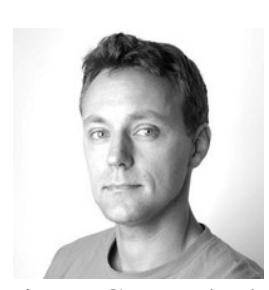

Jesse Williams received his B.S. degree from the University of Pittsburgh in 2001, and his Ph.D. degree from the University of California, Santa Barbara in 2008. He was in the International Center for Materials Nanoarchitectronics at the National Institute for Materials Science, Japan, and was VP of Engineering at LIM Innovations. Currently, he is at Global Technology Connection Inc.

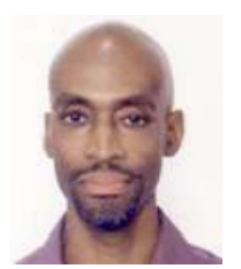

Rodney Martin is a senior researcher in the Intelligent Systems Division and acts as the Deputy Data Sciences Group Lead. Over the course of his $12+$ years at NASA Ames Research Center, he has worked in the application areas of robotics, data mining for aviation safety and space propulsion, among other areas. He acts as the research lead and facility support manager for Sustainability Base, the agency's flagship LEED-platinum certified green building that acts as a living laboratory for testing advanced information and sustainable technologies. Life support and other safety critical systems that will be essential for missions in support of future advanced and fully sustainable exploration habitats can directly benefit from these efforts.

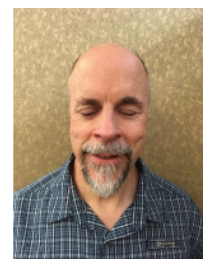

Craig Moore is a member of the Integrated Systems Health Management and Automation branch which is part of the Flight Mechanics and Analysis Division at Marshall Space Flight Center in Huntsville Alabama. For the last 12 years he has supported the branch in its development of ISHM software for the Ares and SLS rockets. For the previous 17 years he has worked in material science as part of the Microgravity Division of the Science Directorate. His work involved theoretical chemistry calculations on novel semiconductor and nonlinear optical materials. He received his M.S. and Ph.D. degrees in Physical Chemistry from the University of Cincinnati and a B.S. in Chemistry from North Dakota State University. 